Trinity University

Digital Commons @ Trinity

Mathematics Faculty Research

Mathematics Department

8-2009

\title{
An Economical Model with Allee Effect
}

Rafael Luís

Saber Elaydi

Trinity University, selaydi@trinity.edu

Henrique Oliveira

Follow this and additional works at: https://digitalcommons.trinity.edu/math_faculty

Part of the Mathematics Commons

\section{Repository Citation}

Luís, R., Elaydi, S., \& Oliveira, H. (2009). An economical model with Allee effect. Journal of Difference

Equations and Applications, 15(8-9), 877-894. http://doi.org/10.1080/10236190802468920

This Post-Print is brought to you for free and open access by the Mathematics Department at Digital Commons @ Trinity. It has been accepted for inclusion in Mathematics Faculty Research by an authorized administrator of Digital Commons @ Trinity. For more information, please contact jcostanz@trinity.edu. 


\title{
An economic Economical Model with Allee effect
}

\author{
Henrique Oliveira \\ Department of Mathematics \\ Instituto Superior Técnico, Technical University of Lisbon, Portugal \\ holiv@math.ist.utl.pt \\ Rafael Luís \\ Center for Math. Analysis, Geometry, and Dynamical Systems \\ Instituto Superior Técnico, Technical University of Lisbon, Portugal \\ rafaelluis@netmadeira.com \\ Saber Elaydi \\ Department of Mathematics, Trinity University \\ San Antonio, Texas, USA \\ selaydi@trinity.edu \\ Lisbon, April 4, 2008
}

\begin{abstract}
The Marx model for the profit rate $r$ depending on the exploitation rate $e$ and on the organic composition of the capital $k$ is studied using a dynamical approach. Supposing both $e(t)$ and $k(t)$ are continuous functions of time we derive a law for $r(t)$ in the long term. Depending upon the hypothesis set on the growth of $k(t)$ and $e(t)$ in the long term, $r(t)$ can fall to zero or remain constant. This last case contradicts the classical hypothesis of Marx stating that the profit rate must decrease in the long term. Introducing a discrete dynamical system in the model and, supposing that both $k$ and $e$ depend on the profit rate of the previous cycle, we get a discrete dynamical system for $r, r_{n+1}=f_{a}\left(r_{n}\right)$, which is a family of unimodal maps depending on the parameter $a$, the exploitation rate when the profit is zero.
\end{abstract}


In this map we can have a fixed point when $a$ is small and, when we increase $a$, we get a cascade of period doubling bifurcations leading to chaos. When $a$ is very big, the system has again periodic stable orbits of period five and period three. But, when we incorporate the Allee effect in the profit rate the system turns completely previsible. Interesting is the fact that we can find in this model the behaviour of the Kondratieff waves in long term economical systems.

Keys Words: Profit rate, organic composition of the capital, exploitation rate, chaos, Allee effect, Kondratieff waves, Stability.

\section{Introduction}

We find in [12] a well known equation from economic theory. In this work we will not only recall it's deduction but also study it in terms of a discrete dynamical systems theory.

The equation quoted above describes the behaviour of the profit rate $r$, with the exploitation rate $e$ and with the organic composition of the capital $k$, in the Marx model. The study of this equation in a dynamical system perspective seems to be very interesting, because it relates the three magnitudes in only one relation:

$$
r=\frac{e}{1+k} .
$$

It's our goal to use this equation as the starting point of a study in which we may model $e(t), k(t)$ and $r(t)$ as variables which depend on $t$, using the formalism of discrete dynamical systems.

In the second section we will present a model formulation considering that the organic composition of the capital and the exploitation rate are dependent on the same magnitudes from the previous cycle. We will have discrete variables and the system starts "to react" to what happened before. In this section we will also introduce a concrete model, that, like many other possible models, may describe the dynamical situation in a realistic way.

In the third section we will study the existence of positive fixed point and their stability, if they exist, and their interpretation in the context of the profit rate. In the next section, we will prove that, in certain cases, this approach model for the profit rate may lead to stability situations with well defined limits, or to periodic orbits (where the profit rate repeats itself from $p$ to $p$ units of time), or to chaos and, in this case, it is impossible to predict 
the evolution of the system, which becomes strongly dependent on the initial conditions. We will use in this section, mathematical tools to prove this behaviour in the profit rate.

In the fifth section, we will study the model for the profit rate with Allee effect. We will prove that when the model has this behaviour the profit rate becomes stable, turning the system completely previsible. We also study the non autonomous case establishing conditions for the Allee point and for the carrying capacity (first and second positive fixed point, respectively). In the last section, we will find, in the model, situations that can explain the kondratieff waves, turning the system very interesting in a long term.

We will prove that the Marx model is open to several mathematical approaches and, depending on which approach we use, the conclusion may go from decrease, to stability, to periodicity and even to chaos.

\section{Model formulation}

To deduct the relation (1.1) let's remember that Marx defined the "organic composition of capital $k$ " as the ratio of what he called constant capital to variable capital. It's important to verify that constant capital is not what we today call fixed capital, but circulating capital, such as raw materials. Marx's "variable capital" is defined as advances to labour, that is, total wage payments, or heuristically, $v=w L$ (where $w$ is wages and $L$ is labour employed). So let's consider that

$$
k=\frac{c}{v},
$$

where $c$ is the constant capital and $v$ is the variable capital.

The profit rate, according to Marx, is defined as

$$
r=\frac{s}{v+c}
$$

where $r$ represent the profit, $s$ is the surplus, and $v+c$ are total advances (constant and variable). The surplus, $s$, is the amount of total output produced above total advances, or $s=y-(v+c)$, where $y$ is total output. It is important to note that for Marx only labor produces surplus value. Marx called the ratio of surplus to variable capital the "exploitation rate $e$ ", that is

$$
e=\frac{s}{v}
$$


(surplus produced for every dollar/euro spent on labor).

Dividing the numerator and the denominator of (2.2) by $v$ we get the relation (1.1).

To study the profit rate in terms of a discrete dynamical system, let $r_{n}$ be the profit rate at the time unit $n$. We suppose that the exploitation rate and the organic composition of the capital depend on the profit rate obtained in the time instant immediately before, that is

$$
e_{n+1}=E\left(r_{n}\right), k_{n+1}=K\left(r_{n}\right) \text {. }
$$

In this case the profit rate depends also on the profit rate of the previous time unit, that is

$$
r_{n+1}=\frac{E\left(r_{n}\right)}{1+K\left(r_{n}\right)} .
$$

The specific model that we propose is based on the following assumptions:

1. We suppose that we haven't had any losses, i.e., when we consider the economy as a whole, we are supposing that a negative profit rate doesn't exist. In fact, we think that in all of the economical activity there exists a positive balance (possibly zero) of the profit. On the other hand, the profit rate can't be unlimited because that contradicts the fact that the total quantity of "money that exists" in the planet is finite.

2. When the profit rate is low, the exploitation tends to increase. On the other hand, when the profit rate is high, the pressure on workers tends to decrease and therefore the exploitation rate tends to decrease to values near zero. So we consider that

$$
E_{a}\left(r_{n}\right)=\frac{a r_{n}}{1+r_{n}^{2}}
$$

is a model for the exploitation rate, where $a>0$ is constant. Note that the function $E_{a}$ has the following properties

$$
E_{a}(0)=E_{a}(\infty)=0, E_{a}^{\prime}(0)=a .
$$

Hence $a>0$ measures the relative growth of the exploitation rate when the profits are low. 


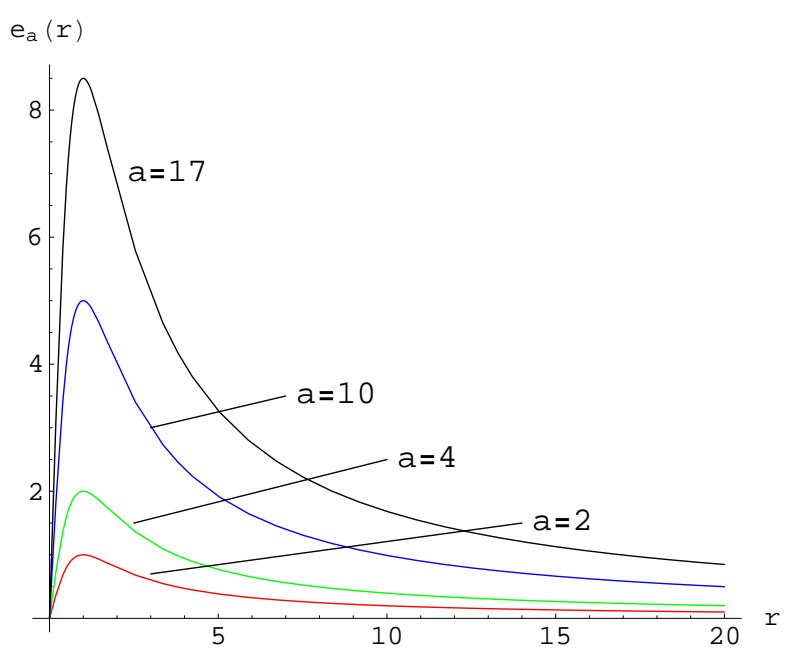

Figure 1: Progress of the exploitation rate

3. When the profit is zero we have a relative amount for investment that decreases as long as the profit increases. If the economical system has a low profit rate, the tendency will be to incorporate more capital (invest) and, on the other hand, to decrease the human capital, through dismissals, that will lead to an increase of the organic composition of the capital. If the profit is very high, the tendency will be to reinvest in the capital: human capital (technical formation) and constant capital (technological innovation). A model that describes this reality is given by an incorporation of the capital function depending on the former profit rate, that is,

$$
K_{b, d}\left(r_{n}\right)=\frac{e^{r_{n}-b}}{\left(r_{n}+d\right)^{2}},
$$

where $b, d>0$ are constant. Note that the function $K_{b, d}$ has the following properties

$$
K_{b, d}(0)=\frac{1}{d^{2} e^{b}}>0, K_{b, d}(\infty)=\infty, K_{b, d}^{\prime}(0)=\frac{d-2}{d^{3} e^{b}} .
$$

This means that $\frac{1}{d^{2} e^{b}}$ gives the initial amount, the organic composition of the capital as a function of the profit, grows unlimitedly, which isn't realistic, but it is used to make the model approachable and $\frac{d-2}{d^{3} e^{b}}$ measures the growth for the organic composition of the capital when the profits are low. 

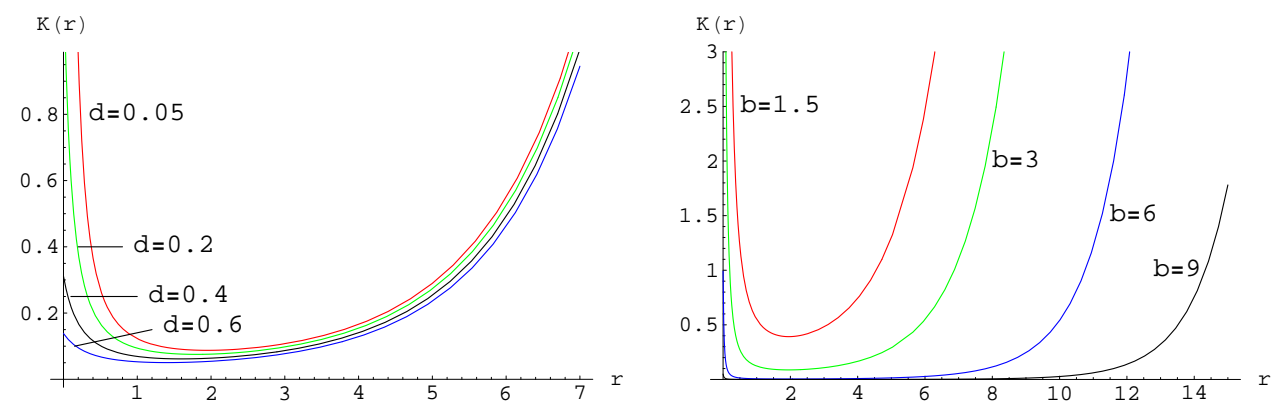

Figure 2: The progress for the organic composition of the capital in the model (2.8). In the left plot we fix the parameter $b$ and variate $d$ in an increasing way, while in the right plot we fix $d$ and variate $b$ in an increasing way. In both cases, in general, the behaviour of the organic composition of the capital is similar. As according $b$ or $d$ increases, for low profits the tendency is decreases the organic composition of the capital, for high profits, the tendency is increases the organic composition of the capital.

By replacing the relations (2.6) and (2.8), the model equation (2.5) for the profit rate becomes

$$
r_{n+1}=\frac{a r_{n}\left(r_{n}+d\right)^{2}}{\left(1+r_{n}^{2}\right)\left[\left(r_{n}+d\right)^{2}+e^{r_{n}-b}\right]} .
$$

Now we are going to study the effect of the parameters $b$ and $d$ in the model (2.8).

When we fix the parameter $b$ and variate $d$ in an increasing way, we observe that the progress of the organic composition of the capital is similar for the different values considered for $d$, as the profits increases. However, the variation of $d$ leads to a decrease of the organic composition of the capital, when the profits are low. This means that the only thing that this parameter $d$ conditions is the initial value for the organic composition of the capital, that is, the initial investment (see the left plot in figure 2).

On the other hand, when we fix the parameter $d$ and variate $b$ in an increasing way we verify that the progress of the organic composition of the capital in model (2.8) as a similar shape for each value considered for $b$. We observe that, in all of this situations for the variation of the parameter $b$, the organic composition of the capital decreases for low values of the profits, until it stabilizes during a specific profits period, and after that it grows according 
to the increasing of the profits. This situation of increasing of the organic composition of the capital begins at a later period according to the parameter $b$ increasing. Note that, when the profits are low, this variation of $b$, to leads a decreasing situation of the initial amount of the organic composition of the capital (see the right plot in figure 2).

All this observations obey to our assumption. So, for a concrete study we need to chose a value for $b$ and $d$. The study that we will do is valid for others values of this two parameters. If we chose other values for the parameters $b$ and $d$, we will only obtain others values for the profits, but the interpretation is the same. So, according to our assumptions we believe that the ideal situation is $b=3$ and $d=\frac{1}{100}$. Note that, the choice of this two values is conditioned by the initial amount for the organic composition of the capital.

We rewrite the model equation (2.10) as

$$
r_{n+1}=\frac{a r_{n}\left(r_{n}+\frac{1}{100}\right)^{2}}{\left(1+r_{n}^{2}\right)\left[\left(r_{n}+\frac{1}{100}\right)^{2}+e^{r_{n}-3}\right]}=f_{a}\left(r_{n}\right) .
$$

We observe that the function $f_{a}(r)$ for all $a>0$ in $r \in[0,+\infty$ [ is continuous, $f_{a}(r) \geq 0, f_{a}(0)=f_{a}(\infty)=0$. The function $f_{a}^{\prime}(r)$ is also continuous and has only one root for all $r \geq 0$. Therefore $f_{a}(r)$ has a unique positive critical point $c \approx 1.10042$ and consequently is superiorly limited. Furthermore, $f_{a}^{\prime}(0)=p a$, where $p \approx 0.00200453$. The origin is a fixed point locally asymptotically stable if $a<\frac{1}{p}$ and unstable if $a>\frac{1}{p}$. When $a=\frac{1}{p}$ we have $f_{a}^{\prime \prime}(0) \approx 397.202$ and consequently instability (see [2]).

\section{Existence of positive fixed point and their stability}

In this section we investigate the existence of positive fixed points for the model (2.11) and their stability, if they exist.

Theorem 3.1 For the model (2.11), there are two positive fixed points if $a>a_{c}$ where $a_{c} \approx 1.64271$, a unique positive fixed point if $a=a_{c}$ and no positive fixed points if $a<a_{c}$. 
Proof. To find the positive fixed point of (2.11) we consider the equation

$$
\frac{a\left(r+\frac{1}{100}\right)^{2}}{\left(1+r^{2}\right)\left[\left(r+\frac{1}{100}\right)^{2}+e^{r-3}\right]}=1
$$

or, equivalently,

$$
\ln \left(1+r^{2}\right)+\ln \left(\left(r+\frac{1}{100}\right)^{2}+e^{r-3}\right)-2 \ln \left(r+\frac{1}{100}\right)=\ln a .
$$

Let $g(r)=\ln \left(1+r^{2}\right)+\ln \left(\left(r+\frac{1}{100}\right)^{2}+e^{r-3}\right)-2 \ln \left(r+\frac{1}{100}\right)$. We have

$$
g^{\prime}(r)=\frac{2 r}{1+r^{2}}-\frac{2}{r+\frac{1}{100}}+\frac{2\left(r+\frac{1}{100}\right)+e^{r-3}}{\left(r+\frac{1}{100}\right)^{2}+e^{r-3}}
$$

Finding the solutions of $g^{\prime}(r)=0$ we have a unique positive critical point $r_{c} \approx 0.478625$ of $g(r)$.

We observe that $g(r)>0$ for all $r \geq 0$, and if $g\left(r_{c}\right)=\operatorname{lna}$ that is $a \approx 1.64271$, there exists a unique positive solutions $r=r_{c}$ to (3.2), if $g\left(r_{c}\right)<\ln a$, there exists two positive solution to (3.2), and if $g\left(r_{c}\right)>\ln a$, there exist no positive solution to (3.2). In figure 3 we can see an example for this conclusion.

Remark 3.2 If $r_{1}$ and $r_{2}$ are two positive fixed points of (2.11) such that $r_{1}<r_{2}$, then $r_{1}<r_{c}<r_{2}$ and $g^{\prime}\left(r_{1}\right)<0=g^{\prime}\left(r_{c}\right)<g^{\prime}\left(r_{2}\right)$

To study the stability of the positive fixed points for the model (2.11), if they exist, we observe that, for the function $f_{a}(r)$ defined in $(2.11)$ we have

$$
\begin{aligned}
f_{a}^{\prime}(r)= & \frac{a\left(r+\frac{1}{100}\right)}{\left(1+r^{2}\right)\left(e^{r-3}+\left(\frac{1}{100}+r\right)^{2}\right)} \\
& {\left[-r\left(r+\frac{1}{100}\right)\left[\frac{e^{r-3}+2\left(\frac{1}{100}+r\right)}{\left(e^{r-3}+\left(\frac{1}{100}+r\right)^{2}\right)}+\frac{2 r}{1+r^{2}}\right]+3 r+\frac{1}{100}\right] }
\end{aligned}
$$

From (3.1) we know that, if $r$ is a positive fixed point, then it satisfies

$$
\frac{a\left(r+\frac{1}{100}\right)^{2}}{1+r^{2}}=\left(r+\frac{1}{100}\right)^{2}+e^{r-3}
$$




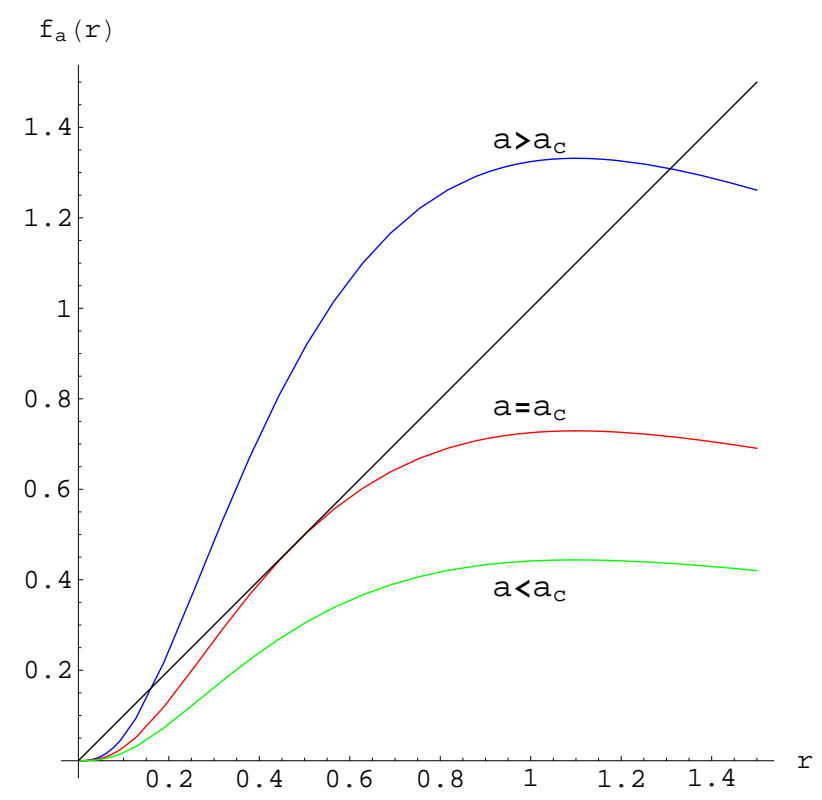

Figure 3: Zero, one and two positive fixed points for the profit rate when $a$ is respectively, less than $a_{c}$, equal $a_{c}$ and greater than $a_{c}$, where $a_{c} \approx 1.64271$

So it follows that

$$
f_{a}^{\prime}(r)=1-r\left[\frac{2 r}{1+r^{2}}-\frac{2}{r+\frac{1}{100}}+\frac{1+r^{2}}{a}\left(\frac{2}{r+\frac{1}{100}}-1\right)+1\right]=1-r g^{\prime}(r)
$$

Therefore $r$ is locally asymptotic stable if

$$
0<r g^{\prime}(r)<2
$$

and is unstable if

$$
r g^{\prime}(r)<0 \text { or } r g^{\prime}(r)>2
$$

To study the stability of each positive fixed point, if they exist, we consider the following two cases:

1. Suppose that the model (2.11) has only one positive fixed point $r=r_{c}$. Then from (3.5) we have $f_{a}^{\prime}\left(r_{c}\right)=1$. Seeing that $f_{a}^{\prime \prime}\left(r_{c}\right) \approx-2.36074 \neq$ 0 then by Saber Elaydi (see [2]) $r=r_{c}$ is unstable. More precisely, $r=r_{c}$ is semi-stable on the right side, if the initial value $\left.r_{0} \in\right] r_{c}, r_{3}[$, 

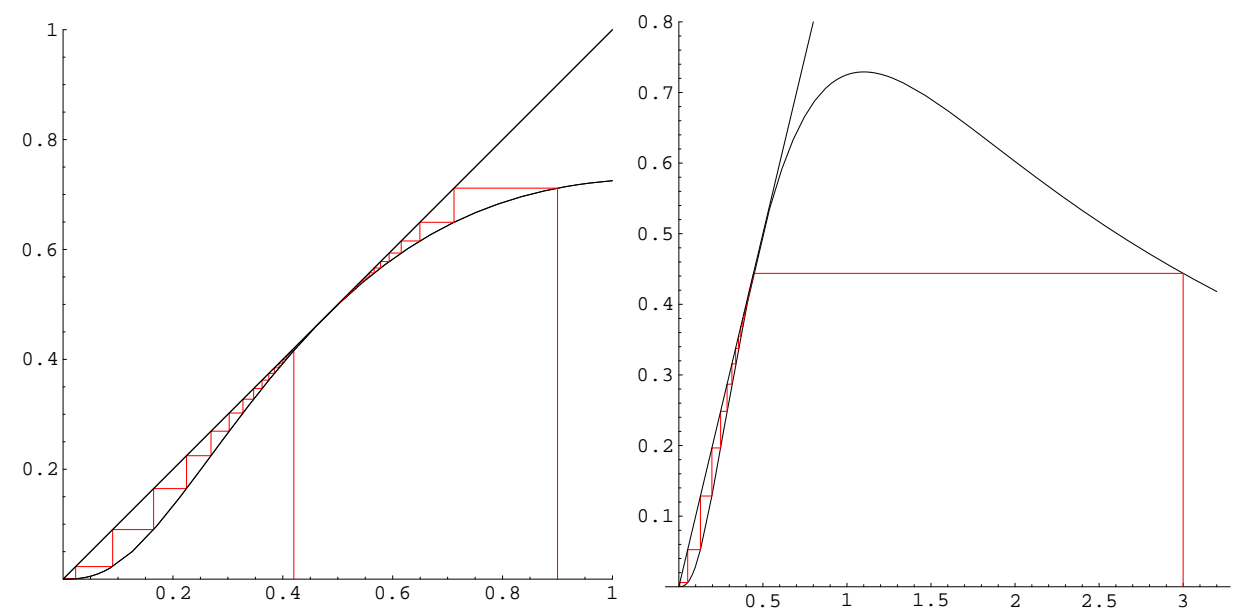

Figure 4: Stability of the unique positive fixed point. In the first case $r=r_{c}$ is semi-stable on the right side now that the initial profit rate $\left.r_{0} \in\right] r_{c}, r_{3}[$ and unstable on the left side since $\left.r_{0} \in\right] 0, r_{c}$ [. In the second case $r=r_{c}$ is unstable now that $\left.r_{0} \in\right] 0, r_{c}[\bigcup] r_{3}, \infty[$

where $f_{a}\left(r_{3}\right)=r_{1}$ and $r_{3}>c$ where $c$ is the critical point of $f_{a}$ determined in the section 2 and $r=r_{c}$ is unstable if the initial value $\left.r_{0} \in\right] 0, r_{c}[\bigcup] r_{3}, \infty[$ (see figure 4$)$.

2. Suppose now that, there exist two positive fixed points for the profit rate $r_{1}$ and $r_{2}$ such that $r_{1}<r_{2}$. It follows from remark 3.2 that $r_{1} g^{\prime}\left(r_{1}\right)<0$ and therefore $r_{1}$ is always unstable (note that $\left.r_{1} \in\right] 0, r_{c}[$ ). For $r_{2}$ we know that $g^{\prime}\left(r_{2}\right)>0$, and therefore $r_{2} g^{\prime}\left(r_{2}\right)>0$. We have two situations:

- $0<r_{2} g^{\prime}\left(r_{2}\right)<2$ if and only if $\left.r_{2} \in\right] r_{c}, r_{s}$ [, where $r_{s} \approx 3.3976$ and consequently $r_{2}$ is locally asymptotic stable,

- $r_{2} g^{\prime}\left(r_{2}\right)>2$ if and only if $\left.r_{2} \in\right] r_{s},+\infty\left[\right.$ and therefore $r_{2}$ is unstable.

In figure 5 we illustrate these ideas.

We summarise this results about the stability of the fixed point in the following theorem.

Theorem 3.3 Consider $r_{c}$ and $a_{c}$ in the same conditions of the proof of theorem 3.1. For $a=a_{c}$ there exists a unique positive unstable fixed point 

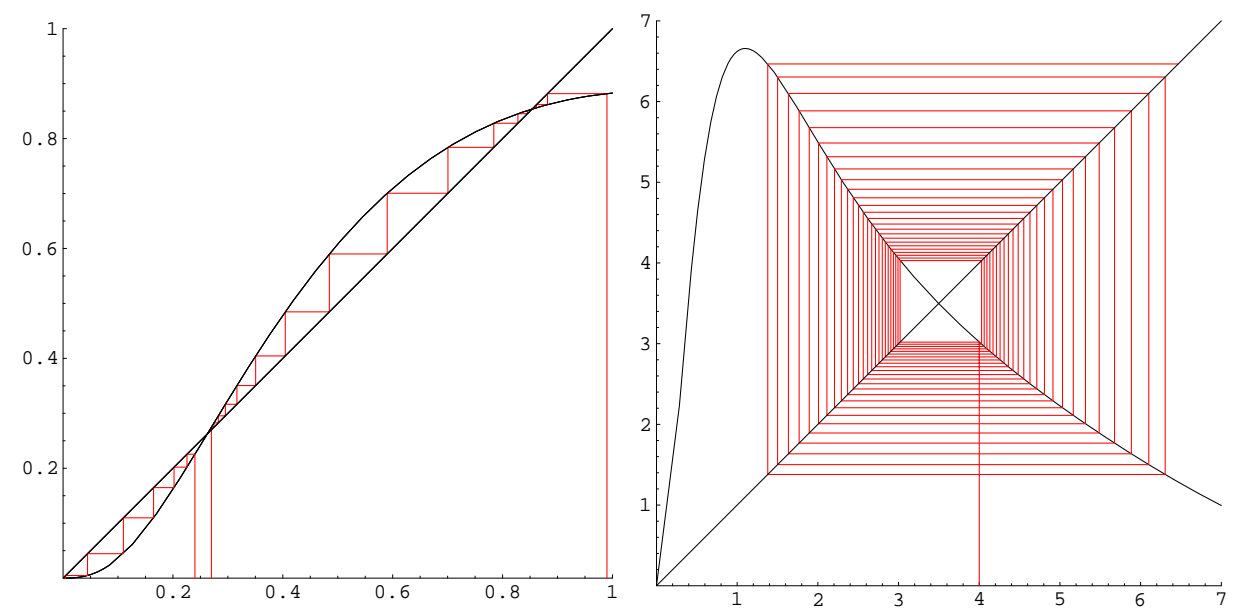

Figure 5: Stability of the two positive fixed points. In the first case $r_{1}$ is unstable and $r_{2}$ is locally asymptotic stable $\left(r_{2} \in\right] r_{c}, r_{s}[)$. In the second case $r_{2}$ is unstable $\left(r_{2} \in\right] r_{s},+\infty[$.

$r=r_{c}$. For $a>a_{c}$ there exists two positive fixed points $r_{1}$ and $r_{2}$ such that $r_{1}<r_{2}$, where $r_{1}$ is always unstable and $r_{2}$ is locally asymptotic stable if $\left.r_{2} \in\right] r_{c}, r_{s}\left[\right.$, where $r_{s} \approx 3.3976$, and is unstable if $\left.r_{2} \in\right] r_{s},+\infty[$.

These ideas, in our model, for the profit rate means that:

- When only exists one positive fixed point, the profit rate can be stable or unstable, after a few units of time. If every initial profit rate $r_{0}$ belongs $] 0, r_{c}[\bigcup] r_{3}, \infty[$ we will obtain profits zero and consequently instability on the initial profit rate. On the other side, if $\left.r_{0} \in\right] r_{c}, r_{3}[$ we will obtain a stable profit after the results.

- When we have two positive fixed points such that $r_{1}<r_{2}$, the profit rate can go to zero, after a few units of time, if every initial profit rate $r_{0}$ is on the left side of the first positive fixed point or can go to the second positive fixed point. So the positive fixed point $r_{1}$ for the profit rate, in this situation, is unstable. Observe that, the second fixed point for the profit rate can be stable or not, depends on the initial profit rate. If the initial profit rate belongs to the limited interval $] r_{c}, r_{s}[$, then after the results we have a stable profit rate, but if the initial profit rate is very high, that is, if it belongs to an unlimited interval ]$r_{s},+\infty[$, then the profit rate goes to zero after the results. 


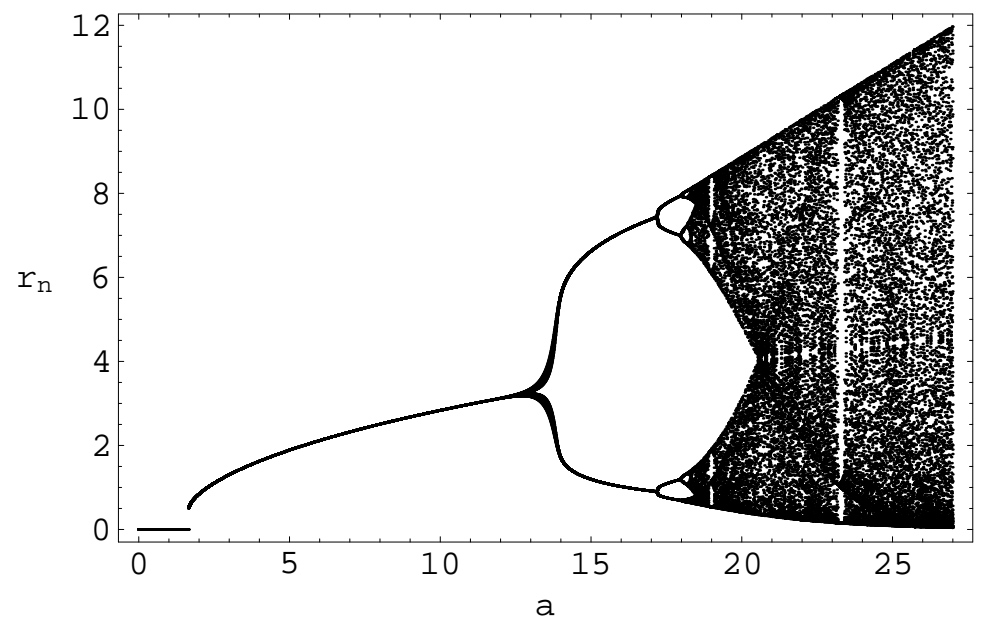

Figure 6: Bifurcation diagram for profit rate where the values of $r_{n}$ are plotted as a function of the parameter "a".

\section{Chaos in the profit rate}

In this section we study the profit rate using mathematical tools from dynamical system theory. We use some concepts of this theory to establish chaos in the profit rate.

When we variate the parameter $a$ in an increasing way, model (2.11) exhibits a typical period doubling bifurcation as we show in figure 6 .

For values $0<a<12.5$, approximately, model (2.11) has a stable equilibrium point. The profit rate tends to stabilise in a specific value. When $12.5<a<17.09957$, approximately, we have a two cycle. From two to two units of time we adjust the exploitation rate and the organic composition of the capital to maximise the profits. As according to the increase of the parameter $a$ we have more cycles for the profit rate with period doubling, such as $4,8,14, \ldots$ This situation of period doubling becomes more complex until the system, for the profit rate, turns completely unpredictable. Surprisingly, after this chaotic period we can find period five orbits, for example for $a=21.94$. This means that, from five to five units of time the profit rate has five different values. When the parameter $a$ grows even more, we have again more aperiodic situations until we fall in a periodic three zone. This situation happens for values $23.082565<a<23.217854$, approximately. This means that we have a triennial repetition for the profit rate. 
This situation of apparent stability inside chaos will coexist with infinity unstable orbits of all periods, as is guarantied by Sharkovsky theorem [10].

In [5] Jia Li e al proved that if all $2^{i}-$ cycles, $i=0, \ldots, n-1$, becomes unstable as the parameter $a$ variates, then there exist at least $2^{n}$ locally asymptotically stable $2^{n}$-cycles. In our model, this means that even that all $\left|f_{n}^{2^{i}}(r)\right|>1, i=0, \ldots, n-1$, we have at least $2^{n}$ locally asymptotically stable $2^{n}$-cycles for the profit rate. So after a long unstable period for what we can not predict nothing, the system exhibits stability leading the profits to stabilise.

The Lyapunov exponent is a mathematical indicator of the exponential degree of the velocity according to which two arbitrary nearby orbits grow further apart as the number of iterations increases. We can define it as following:

Definition 4.1 The Lyapunov exponent $\lambda\left(r_{0}\right)$ for a point $r_{0}$ is given by

$$
\lambda\left(r_{0}\right)=\lim _{n \rightarrow+\infty} \sup \frac{1}{n} \sum_{k=0}^{n-1} \ln \left|f^{\prime}\left(r_{k}\right)\right|
$$

where $r_{k}=f_{a}^{k}\left(r_{0}\right)$.

In practice, to calculate experimentally the value of the Lyapunov exponent we can use the formula

$$
\lambda\left(r_{0}\right)=\lim _{n \rightarrow+\infty} \frac{1}{n} \sum_{k=0}^{n-1} \ln \left|f^{\prime}\left(r_{k}\right)\right|,
$$

that is equivalent to

$$
\lambda\left(r_{0}\right)=\ln \lim _{n \rightarrow+\infty} \sqrt[n]{\left|\left(f^{n}\left(r_{0}\right)\right)^{\prime}\right|} .
$$

If the absolute value of $f^{\prime}\left(r_{k}\right)$ is greater than one, then the Lyapunov exponent is positive, which implies a sensitive dependence of the initial conditions imposed [2].

For our model, if $r_{0}=2$ we have $r_{k} \approx 3.62895$ and $\left|f_{25}^{\prime}\left(r_{k}\right)\right| \approx-1.64073$, then by (4.2), we have

$$
\lambda(2)=\lim _{n \rightarrow+\infty} \frac{1}{n} \sum_{k=0}^{n-1} \ln 1.64073 \approx 0.4951,
$$




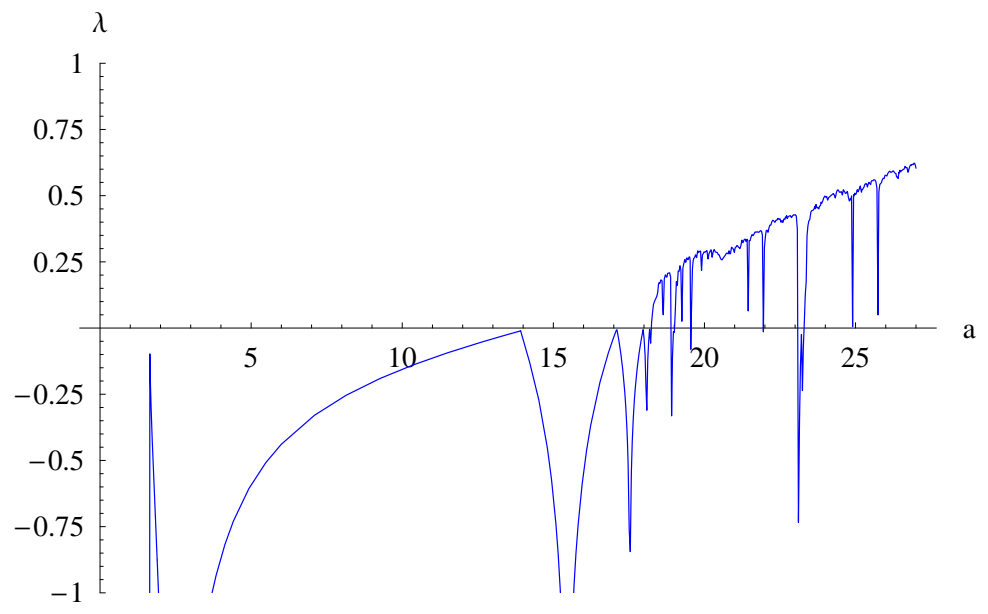

Figure 7: Lyapunov exponent $\lambda$ of the function $f_{a}$ under the parameter $a$ applied to the profit rate

which implies that the point $x=2$ for $f_{25}$ has a sensitive dependence on the initial conditions.

In figure 7 we can see the progress of the Lyapunov exponent of the function $f_{a}$ under the parameter $a$. We observe that, for model (2.11), we have sensitive dependence on the initial profit rate, seeing that, the Lyapunov exponent becomes positive according the variation of the parameter $a$ (values of $a$ greater than 18.2).

Another mathematical tool to indicate chaos is the topological entropy. To introduce this concept we will use the kneading theory [9]. For this it is necessary to define the growth number $s$ for unimodal maps (a function that has only one critical point).

Definition 4.2 The lap number, $l\left(f_{a}^{n}\right)$, is the number of maximal intervals of monotonicity of $f_{a}^{n}$ ( $f_{a}^{n}$ is piecewise-monotone). The growth number $s$ is obtained by the relation

$$
s\left(f_{a}\right)=\lim _{n \rightarrow+\infty}\left(l\left(f_{a}^{n}\right)\right)^{\frac{1}{n}} .
$$

When the growth of the laps is small (polynomial with the number of iterates) we do not have chaos, but when the growth of the lap number is 
exponential (the system responds in a more sensitive way to the precedent cycle data), we have chaos. This happens when the growth number is greater than 1.

To calculate the topological entropy, we define the parity function for the turning point $c \approx 1.10042$ as follows:

Definition 4.3 The parity function for the turning point $c$ is given by

$$
\varepsilon(x)=\left\{\begin{array}{l}
1 \text { if } x<c \\
0 \text { if } x=c \\
-1 \text { if } x>c
\end{array}\right.
$$

It follows a few results of these two definitions:

Theorem 4.4 1. The kneading determinant is a formal series in $t$ given by

$$
Z(t, a)=1+\sum_{n=1}^{\infty}\left(\prod_{j=1}^{n} \varepsilon\left(f_{a}^{n}(c)\right) \cdot t^{n}\right)
$$

2. In case of periodic orbits of $c, Z(t, a)$ is a polynomial of degree $(n-1)$. The inverse of the minor root of $Z(t, a)$ in $[0,1]$ is the growth number of $f_{a}$.

3. The topological entropy $h_{t}$ is given by the relation $h_{t}=\log _{2}(s)$.

Proof. See [9]

Our function has two intervals of monotonocity, that is, $f_{a}$ increases in ] $0, c[$ and it decreases in $] c,+\infty[$. For $a=25$ we have that, the first terms of the kneading polynomial are

$$
1-t-t^{2}-t^{3}+t^{4}-t^{5}+t^{6}-t^{7}+t^{8}-t^{9}+t^{10}-t^{11}+\ldots
$$

The smallest real root of this polynomial belongs to $[0,1]$ (with an approximation of 16 decimal digits for the polynomial of degree 55), it is approximately equal to 0.562781 . The topological entropy therefore is given by

$$
h_{t}=\log _{2}\left(0.562781^{-1}\right) \approx 0.83064 \text {. }
$$

In figure 8 we can see the evolution of the topological entropy values for the profit rate. 


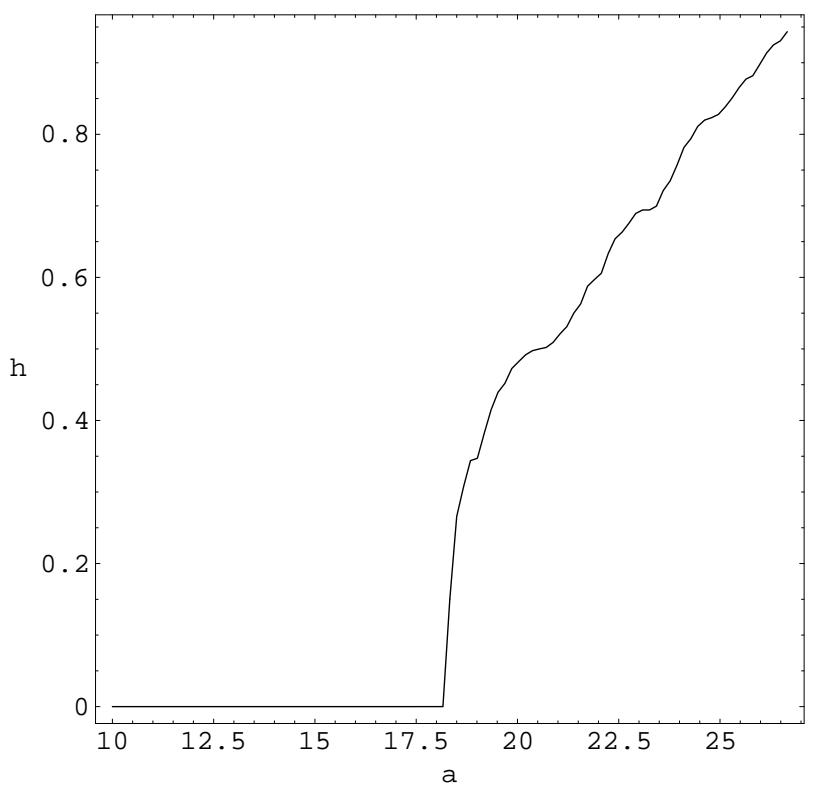

Figure 8: Progress of the topological entropy $h_{t}$ for the model (2.11). According the growth of the parameter $a$ the topological entropy $h_{t}$ increase for values near 1.

The fact that the topological entropy increases and is greater than zero (associated to the fact that the Lyapunov exponent is positive) means that the dynamical system becomes more complex according to the growth of the parameter $a$. For values of $a>18.2$ the model starts to exhibit chaos, something that results apparently in the bifurcation diagram when we close the classical duplication of the period process, where we can see clearly in this diagram an aperiodic band with no stability. From the economical point of view this situation would also result in a huge complexity and instability of the system. This situation happens when the exploitation rate is very high and the profit rate is very low.

In this model, trying to correct low profit at the cost of high exploitation rate leads inevitably to instability and to chaos, both from the mathematical point of view (mathematical definition of chaos) and from the common sense point of view (economical chaos). For values of $a>18.2$ our model can be unrealistic in the short term, because in those circumstances, after a high profit, we will have a low profit. This variation of the profit rate can be 
explained by some factors that happen in an economy as a whole, like wars, natural catastrophes, pressures of various agents, etc. If it was possible to introduce an exploitation rate so high that we would be led to this situation, that would mean that the system would react by presenting a reasonable profit rate from five to five or three to three units of time (values of $a$ near to 21.94 , in the first case, and 23.1 in the second case).

We believe that the exploitation rate must be kept below a certain critical value, which in our model, is for values $a<12.5$, to maintain the stability of the system in a constant profit rate along the economic cycles.

\section{Allee effect in the profit rate}

In this section we study the profit rate when we incorporate the Allee effect in the model (2.11). We start defining the following concept.

Definition 5.1 Every model is under Allee effect whenever $f_{a}(r)$ support three fixed point, 0 and two positive fixed point, $r_{1}$ and $r_{2}$, such that $r_{1}<r_{2}$ and 0 is globally attracting in $\left[0, r_{1}\left[, r_{1}\right.\right.$ is a repellor and a positive attractor exists in the interval $] r_{1}, \max _{1}\left[\right.$ where $I_{1}=f_{a}\left(\left[0, r_{2}\right]\right)$.

We call the smaller positive fixed point the Allee point and the greater positive fixed point the carrying capacity.

The model (2.11) for the profit rate is under Allee effect when $a \in] a_{c}, r_{s}[$, where $a_{c} \approx 1.64271$ and $r_{s} \approx 3.3976$, now that, for these values the origin is globally attracting, the first positive fixed point is repellor and the second positive fixed point is attractor.

We proved in section 4 that, for these values of the parameter $a$, model (2.11) has a stable profit rate. So when we incorporate the Allee effect in the profit rate in a long term, the system turns the profit rate stable. In other words, this means that the Allee effect is a good stabiliser as we get to know the results of the profits. This shows that, whenever the model is under the Allee effect, we can predict the variation of the profits leading us, in a safe way, to investment.

In this study we limited our analysis to the autonomous case in which the parameter $a$ is assumed to be independent of time. For us it would be interesting to extend the study to the case when the parameter $a$ is time dependent. One may start by assuming that $a$ is periodic. For this study we 

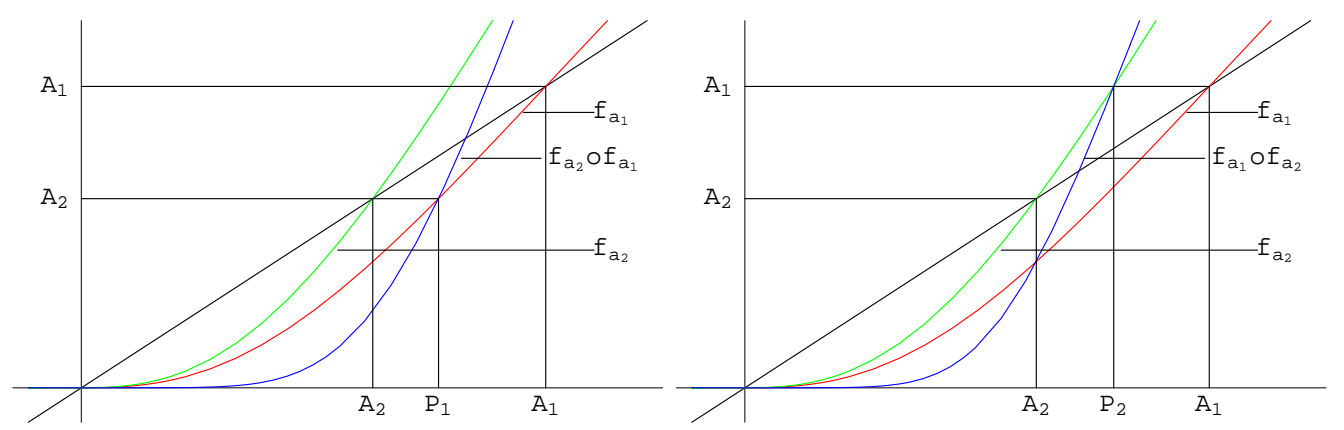

Figure 9: In the left plot we represent the first pre-image of $A_{2}$ by the function $f_{a_{1}}$ while the right plot we represent the first pre-image of $A_{1}$ by the map $f_{a_{2}}$

can compose two maps obtained from (2.11) and study the properties of the fixed point of the two possible composition.

Let's rewrite model (2.11) as $f_{a}(x)=a g(x)$. We verify that $g(x)<$ $x, \forall x \in \mathbb{R}^{+}$. Let $\left.a_{1}, a_{2} \in\right] a_{c}, r_{s}\left[=J\right.$ such that $a_{1}<a_{2}$. Then $f_{a_{1}}(x)<$ $f_{a_{2}}(x), \forall x \in \mathbb{R}^{+}$. Let $A_{1}$ and $A_{2}$ be the Allee points of $f_{a_{1}}$ and $f_{a_{2}}$, respectively, and $K_{1}$ and $K_{2}$ the carrying capacity of this two maps, respectively, i.e.,

$$
\begin{aligned}
& f_{a_{1}}\left(A_{1}\right)=a_{1} g\left(A_{1}\right)=A_{1}, f_{a_{1}}\left(K_{1}\right)=a_{1} g\left(K_{1}\right)=K_{1} \\
& f_{a_{2}}\left(A_{2}\right)=a_{2} g\left(A_{2}\right)=A_{2}, f_{a_{2}}\left(K_{2}\right)=a_{2} g\left(K_{2}\right)=K_{2}
\end{aligned}
$$

The order relation of this two fixed point is $A_{2}<A_{1}<K_{1}<K_{2}$.

We also are interested in study the properties of the Allee points of the composition of the maps $f_{a_{1}}$ and $f_{a_{2}}$. We call the region under study the Allee region, that is, the square with side length $A_{1}$ (the bigger of the Allee points).

Let $P_{1}$ and $P_{2}$ be the first pre-image of $A_{2}$ and $A_{1}$, by the function $f_{a_{1}}$ and $f_{a_{2}}$, respectively,. In other words $\left.\exists P_{1}, P_{2} \in\right] A_{2}, A_{1}\left[: f_{a_{1}}\left(P_{1}\right)=A_{2}\right.$ and $f_{a_{2}}\left(P_{2}\right)=A_{1}$. Consequently, $f_{a_{2}} o f_{a_{1}}\left(P_{1}\right)=A_{2}$ and $f_{a_{1}} o f_{a_{2}}\left(P_{2}\right)=A_{1}$ (see figure 9$)$.

Remark 5.2 We note that both $f_{a_{1}}$ and $f_{a_{2}}$ are homeomorphism in the Allee region.

Theorem 5.3 $f_{a_{2}} o f_{a_{1}}$ and $f_{a_{1}} o f_{a_{2}}$, both, have Allee points, that we denote by $A_{f_{a_{2}} o f_{a_{1}}}$ and $A_{f_{a_{1} o f_{2}}}$, respectively. Moreover $P_{1}<A_{f_{a_{2}} o f_{a_{1}}}<A_{1}$ and $A_{2}<A_{f_{a_{1}} o f_{a_{2}}}<P_{2}$. 
Proof. First we will prove the existence of $A_{f_{a_{2}} \circ f_{a_{1}}}$ and $A_{f_{a_{1}} \circ f_{a_{2}}}$. For $x \in$ $\left[0, A_{2}\right]$ we have $f_{a_{1}}(x)<x$, this implies that $f_{a_{2}}\left(f_{a_{1}}(x)\right)<x$. On other hand when $x \in\left[A_{1}, C\left[\right.\right.$ where $C=\min \left\{C_{f_{a_{1}}}, C_{f_{a_{2}}}\right\}, C_{f_{a_{1}}}$ and $C_{f_{a_{2}}}$ are, respectively, the critical points of $f_{a_{1}}$ and $f_{a_{2}}$, we have that $f_{a_{1}}>x$ and consequently $f_{a_{2}}\left(f_{a_{1}}(x)\right)>x$. Therefore there exists $\left.y \in\right] A_{2}, A_{1}[$ such that $f_{A_{2}} o f_{A_{1}}(y)=y$, i.e., $A_{2}<A_{f_{a_{2}} o f_{a_{1}}}<A_{1}$. In same way we prove that $A_{2}<A_{f_{a_{1}} o f_{a_{2}}}<A_{1}$.

To prove that $\left.A_{f_{a_{2} o a_{a_{1}}}} \in\right] P_{1}, A_{1}$ [ first we will prove that $A_{f_{a_{2}} \circ f_{a_{1}}} \notin$ $\left[A_{2}, P_{1}\right]$. Since that $f_{a_{1}}\left(A_{2}\right)<A_{2}$ we get $f_{a_{2}} \circ f_{a_{1}}\left(A_{2}\right)<A_{2}$, when $x \in$ ] $A_{2}, P_{1}$ [ we have $f_{a_{2}} \circ f_{a_{1}}(x)<x$, and $f_{a_{2}} \circ f_{a_{1}}\left(P_{1}\right)=A_{2}<P_{1}$. Therefore $A_{f_{a_{2}} o f_{a_{1}}} \notin\left[A_{2}, P_{1}\right]$.

Now let $x \in] P_{1}, A_{1}\left[\right.$. By one side we have $f_{a_{2}} o f_{a_{1}}\left(A_{1}\right)=f_{a_{2}}\left(A_{1}\right)>A_{1}>$ $x$ and on other side $f_{a_{2}} o f_{a_{1}}\left(P_{1}\right)=A_{2}<x$, consequently

$$
\exists y \in] P_{1}, A_{1}\left[: f_{a_{2}} o f_{a_{1}}(y)=y=A_{f_{a_{2}} o f_{a_{1}}} .\right.
$$

Following the same reasoning we prove that $A_{2}<A_{f_{a_{1}} o f_{a_{2}}}<P_{2}$.

Remark $5.4 f_{a_{1}}$ and $f_{a_{2}}$ are two maps convex and diffeomorphism with class $C^{2}$. Moreover, in the interval $J$ we have $f_{a_{1}}^{\prime}<f_{a_{2}}^{\prime}\left(a_{1} g^{\prime}(x)<a_{2} g^{\prime}(x)\right.$, $\forall x \in J)$.

Theorem 5.5 If $g^{\prime}\left(A_{2}\right) \geq \frac{1}{a_{1}}+\frac{1}{a_{2}}$ then $P_{1} \geq P_{2}$. Moreover $A_{f_{a_{2}} \text { of } a_{1}} \geq$ $A_{f_{a_{1}} \circ f_{a_{2}}}$.

Proof. Suppose that $A_{1}-A_{2}=\varepsilon>0$ and $f_{a_{2}}(x)-f_{a_{1}}(x)=\delta(x), \forall x \in J$ ( $\delta(x)$ is increase). We need to prove that the first pre-image of $A_{2}$ and $A_{1}$, both, satisfy the relation $P_{1} \geq P_{2}$ or $f_{a_{2}}^{-1}\left(A_{1}\right) \leq f_{a_{1}}^{-1}\left(A_{2}\right)$, that is equivalent

$$
A_{1} \leq f_{a_{2}} o f_{a_{1}}^{-1}\left(A_{2}\right)
$$

By Taylor's series we know that

$$
\begin{aligned}
f_{a_{1}}^{-1}\left(A_{2}\right) & =f_{a_{1}}^{-1}\left(A_{1}-\varepsilon\right) \\
& =f_{a_{1}}^{-1}\left(A_{1}\right)-\left(f_{a_{1}}^{-1}\left(A_{1}\right)\right)^{\prime} \varepsilon+O\left(\varepsilon^{2}\right) \\
& =A_{1}-\frac{\varepsilon}{f_{a_{1}}^{\prime}\left(A_{1}\right)}+O\left(\varepsilon^{2}\right) .
\end{aligned}
$$

Substituting the previous relation in (5.1) we get 


$$
A_{1} \leq f_{a_{2}}\left[A_{1}-\frac{\varepsilon}{f_{a_{1}}^{\prime}\left(A_{1}\right)}+O\left(\varepsilon^{2}\right)\right]
$$

and by Taylor's series again

$$
A_{1} \leq f_{a_{2}}\left(A_{1}\right)-\frac{f_{a_{2}}^{\prime}\left(A_{1}\right)}{f_{a_{1}}^{\prime}\left(A_{1}\right)} \varepsilon+O\left(\varepsilon^{2}\right)
$$

that is

$$
\begin{gathered}
A_{1} \leq a_{2} g\left(A_{1}\right)-\frac{a_{2} g^{\prime}\left(A_{1}\right)}{a_{1} g^{\prime}\left(A_{1}\right)} \varepsilon+O\left(\varepsilon^{2}\right) \\
\Leftrightarrow \frac{a_{2}}{a_{1}} \varepsilon \leq a_{2} g\left(A_{1}\right)-a_{1} g\left(A_{1}\right)+O\left(\varepsilon^{2}\right)=\delta+O\left(\varepsilon^{2}\right),
\end{gathered}
$$

and consequently

$$
\frac{a_{2}}{a_{1}} \varepsilon \leq \delta+O\left(\varepsilon^{2}\right)
$$

So relation (5.2) is equivalent to relation (5.1).

We now that $g^{\prime}\left(A_{2}\right)<\frac{g\left(A_{1}\right)-g\left(A_{2}\right)}{A_{1}-A_{2}}=g^{\prime}(M)<g^{\prime}\left(A_{1}\right)$, where $M \in$ ]$A_{2}, A_{1}[$. So

$$
\begin{aligned}
a_{2} g^{\prime}(M) & =\frac{a_{2} g\left(A_{1}\right)-a_{1} g\left(A_{1}\right)+a_{1} g\left(A_{1}\right)-a_{2} g\left(A_{2}\right)}{A_{1}-A_{2}} \\
& =\frac{f_{a_{2}}\left(A_{1}\right)-f_{a_{1}}\left(A_{2}\right)+A_{1}-A_{2}}{A_{1}-A_{2}}=\frac{\delta+\varepsilon}{\varepsilon} .
\end{aligned}
$$

Therefore $g^{\prime}\left(A_{2}\right)<g^{\prime}(M)<\frac{\delta+\varepsilon}{a_{2} \varepsilon}$.

By hypothesis we have

$$
g^{\prime}\left(A_{2}\right) \geq \frac{1}{a_{1}}+\frac{1}{a_{2}} \Leftrightarrow \frac{1}{a_{1}}+\frac{1}{a_{2}} \leq g^{\prime}\left(A_{2}\right)+O(\varepsilon),
$$

and consequently

$$
\frac{1}{a_{1}}+\frac{1}{a_{2}} \leq \frac{\delta+\varepsilon}{a_{2} \varepsilon}+O(\varepsilon) .
$$

Multiplying both sides of the last relation by $a_{2} \varepsilon$ we get

$$
\frac{a_{2}}{a_{1}} \varepsilon+\varepsilon \leq \delta+\varepsilon+a_{2} \varepsilon O(\varepsilon)
$$

and therefore

$$
\frac{a_{2}}{a_{1}} \varepsilon \leq \delta+O\left(\varepsilon^{2}\right),
$$


that is equivalent to relation (5.2) and therefore the relation $P_{1} \geq P_{2}$ is proved.

Once that $P_{1} \geq P_{2}$ and from theorem 5.3 it follows that $A_{f_{a_{2}} o f_{a_{1}}} \geq$ $A_{f_{a_{1} o f_{a_{2}}}}$.

Now we will focus our attention in the carrying capacity of the composition of the two maps $f_{a_{1}}$ and $f_{a_{2}}$. We know that $\lim _{x \rightarrow+\infty} g(x)=0$, therefore $\lim _{x \rightarrow+\infty} f_{a_{2}} o f_{a_{1}}(x)=\lim _{x \rightarrow+\infty} a_{2} g\left(a_{1} g(x)\right)=0$. Consequently, $\exists S>K_{2}$ : $f_{a_{2}} o f_{a_{1}}(S)=A_{1}<K_{1}$.

Theorem $5.6 f_{a_{2}} o f_{a_{1}}$ has a carrying capacity greater than the Allee point, that we denote by $K_{f_{a_{2}} \text { of } a_{1}}$, such that $\left.K_{{f_{2}}_{2} o f_{a_{1}}} \in\right] K_{1}, S\left[\right.$. Moreover, if $g\left(\frac{a_{1}}{a_{2}} K_{2}\right)<$ $\frac{K_{2}}{a_{2}}$ then $\left.K_{f_{a_{2}} o f_{a_{1}}} \in\right] K_{1}, K_{2}\left[\right.$ and if $g\left(\frac{a_{1}}{a_{2}} K_{2}\right)>\frac{K_{2}}{a_{2}}$ then $\left.K_{f_{a_{2}} o f_{a_{1}}} \in\right] K_{2}, S[$.

Proof. By one side, we have $f_{a_{2}} o f_{a_{1}}\left(K_{1}\right)=a_{2} g\left(a_{1} g\left(K_{1}\right)\right)=a_{2} g\left(K_{1}\right)=$ $\frac{a_{2}}{a_{1}} a_{1} g\left(K_{1}\right)=\frac{a_{2}}{a_{1}} K_{1}>K_{1}\left(a_{1}<a_{2}\right)$, on other side, $\exists S>K_{2}: f_{a_{2}} o f_{a_{1}}(S)=$ $A_{1}<K_{1}$. Consequently, $\left.\exists q \in\right] K_{1}, S\left[: f_{a_{2}} o f_{a_{1}}(q)=q=K_{f_{a_{2}} o f_{a_{1}}}\right.$.

To prove the second part of the theorem we note that from $g\left(\frac{a_{1}}{a_{2}} K_{2}\right)<$ $\frac{K_{2}}{a_{2}}$ follows $f_{a_{2}} o f_{a_{1}}\left(K_{2}\right)<K_{2}$ and therefore $\left.K_{f_{a_{2}} o f_{a_{1}}} \in\right] K_{1}, K_{2}[$. When $g\left(\frac{a_{1}}{a_{2}} K_{2}\right)>\frac{K_{2}}{a_{2}}$ it follows that $f_{a_{2}} o f_{a_{1}}\left(K_{2}\right)>K_{2}$, so $\left.K_{f_{a_{2}} o f_{a_{1}}} \in\right] K_{2}, S[$.

Remark 5.7 The second positive fixed point of $f_{a_{2}} o f_{a_{1}}$ can be greater than the greater fixed point of $f_{a_{2}}$ or not, but it will never be less than the greater fixed point of $f_{a_{1}}$.

Theorem $5.8 f_{a_{1}} o f_{a_{2}}$ has a carrying capacity greater than the Allee point, that we denote by $K_{f_{a_{1}} \circ f_{a_{2}}}$, such that $\left.K_{f_{a_{1}} \circ f_{a_{2}}} \in\right] A_{1}, K_{2}[$. Moreover, if $g\left(\frac{a_{2}}{a_{1}} K_{1}\right)<\frac{K_{1}}{a_{1}}$ then $\left.K_{f_{a_{1}} \text { of } a_{2}} \in\right] K_{1}, K_{2}\left[\right.$ and if $g\left(\frac{a_{2}}{a_{1}} K_{1}\right)>\frac{K_{1}}{a_{1}}$ then $K_{f_{a_{1}} \circ f_{a_{2}}} \in$ ]$A_{1}, K_{1}[$.

Proof. By one side we have $f_{a_{1}} o f_{a_{2}}\left(K_{2}\right)=a_{1} g\left(K_{2}\right)=\frac{a_{1}}{a_{2}} a_{2} g\left(K_{2}\right)=\frac{a_{1}}{a_{2}} K_{2}<$ $K_{2}$ and other side we know that $f_{a_{2}}\left(A_{1}\right)>A_{1}$ that implies $f_{a_{1}} o f_{a_{2}}\left(A_{1}\right)>$ $f_{a_{1}}\left(A_{1}\right)=A_{1}$. Consequently $\left.\exists q \in\right] A_{1}, K_{2}\left[: f_{a_{1}} o f_{a_{2}}(q)=q=K_{f_{a_{1}} o f_{a_{2}}}\right.$.

The second part of the theorem is easily obtained because from $g\left(\frac{a_{2}}{a_{1}} K_{1}\right)<$ $\frac{K_{1}}{a_{1}}$ follows $f_{a_{1}} o f_{a_{2}}\left(K_{1}\right)>K_{1}$ and therefore $\left.K_{f_{a_{1}} o f_{a_{2}}} \in\right] K_{1}, K_{2}[$. The other 


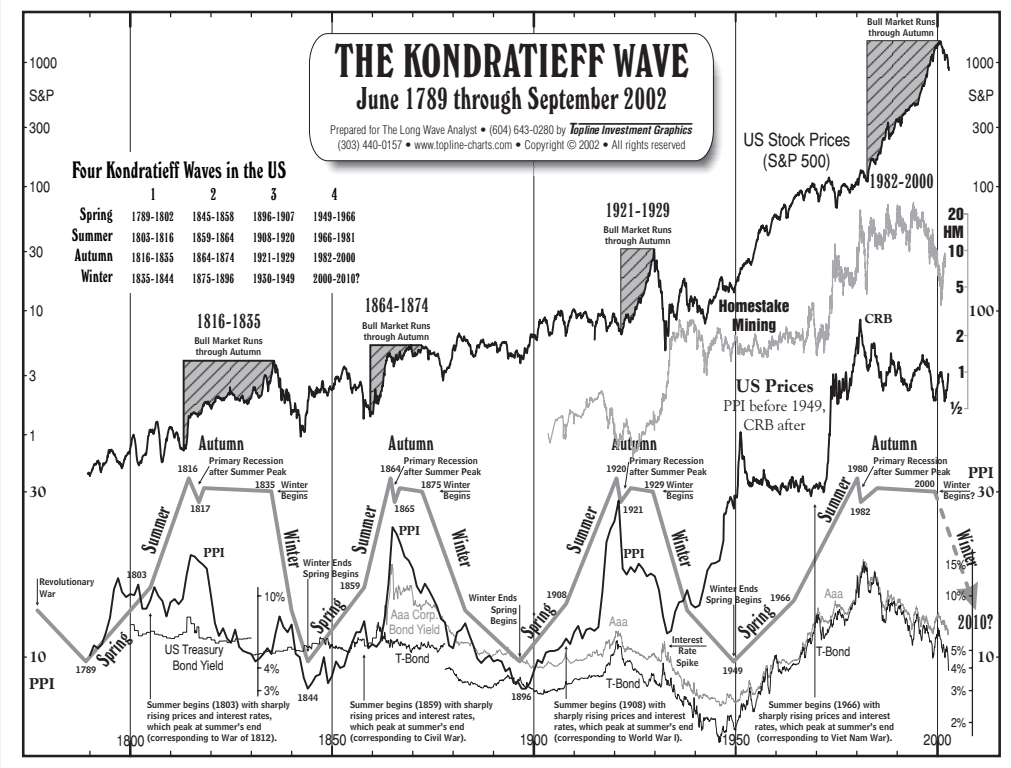

Figure 10: Source: www.pointfigure.dk/filer/dl/kondratieff-wave.pdf, in 3rd of July 2007.

case we get $f_{a_{1}} \circ f_{a_{2}}\left(K_{1}\right)<K_{1}$ that permit to conclude $\left.K_{f_{a_{1}} \circ f_{a_{2}}} \in\right] A_{1}, K_{1}[$.

Remark 5.9 The second positive fixed point of $f_{a_{1}} o f_{a_{2}}$ can be greater than the greater fixed point of $f_{a_{1}}$ or not, but it will never be greater than the greater fixed point of $f_{a_{1}}$.

\section{Kondratieff waves in the profit rate}

If the time unit is long (a decade, a quarter of century, etc), model (2.11) can be very interesting for the economic cycles prevision for the long term, as are the Kondratieff waves [6].

"Kondratieff waves may be defined as a pattern of regularity characteristic of structural change in the modern world economy". More and less 50 years for each cycle, "it consists of an alternation of periods of high sectoral growth with others, start-up periods of slower growth. The study of this pattern helps to trace the evolution of the global economy, and aids in politicoeconomic prediction." 


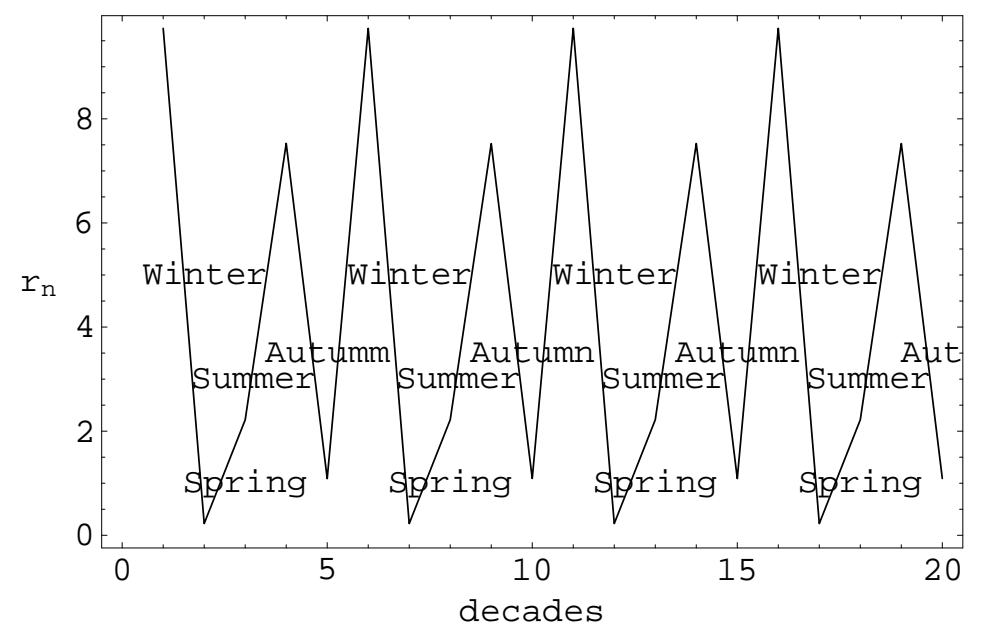

Figure 11: For $a=21.94$ model (2.11) can be interpreted like the Kondratieff waves. The progress for the profit rate can be looked in four seasons that repeat each five decades.

"A Kondratieff wave consists of four distinct phases, or distinguishable, dramatic mood changes, the tone of which determines the actions of individuals involved in the economy. The awareness of these characteristics allows for the anticipation of the change in the economy and the psychological mood that will prevail". So for these four distinct phases in the Kondratieff waves some analysts have compared them to the seasons: spring (inflationary growth, expansion), summer (stagflation, recession), autumn (deflationary growth, plateau) and winter (depression), that changes each fifty years (figure 10).

For example, when $a=21.94$ we have one situation, for model (2.11), near to the Kondratieff waves (figure 11). If we establish a parallel between the Kondratieff waves and the progress of the profit rate, that we represent in figure 11, we observe a similar situation in the line. However the Autumn's situation doesn't permit to infer any conclusions, because we have first a fall in the profit rate and after that an accelerated growth until the beginning of the winter. This situation isn't similar to the Kondratieff waves because there, after the first fall there is a little growth after that, a slow fall until winter. We believe that this situation in our model is originated by the fact that when the exploitation rate grows, it origins a strong influence in the profit rate leading to this abrupt variation in the autumn's season. However, 
this situation can be adjusted to more realistic values.

\section{Conclusion}

The problem of the decreasing of the profit rate with time has been the subject of multiple discussions in the last 150 years.

In this applied mathematical study, we used the equation that arises from economic theory, a purely static equation, that only gives us the fixed relation between the profit rate, the exploitation rate and the organic composition of the capital, as a starting point for several dynamical considerations.

It's obvious that we can't infer conclusions about the profit rate evolution if we regard this equation as a relation between constants. This is what has been done by Human Science in the past, as they wanted to obtain results about the profit rate evolution in a mid or a long term period, when the model was based on only a relation between fixed magnitudes, variables that aren't even totally independent themselves.

Our goal, as mathematicians, was to be able to construct an evolutive model in time, that starts from the identified equation to give it a dynamical interpretation.

We introduced a dynamical system, unidimensional, that reacts to the previous cycle of the profit rate, trying to reply by varying the organic composition of the capital by one side: dismissing when the profits are low, buying equipment (reinvesting) when the profits are high; and increasing the exploitation rate when the profits are low in the previous cycle. We obtain a unimodal system that iterated is, in our opinion, a lot more realistic than one blind prediction based only on immutable temporary laws, or in linear approaches, than are unreal in a long term.

When the system doesn't try to react desperately to low profits in the previous cycle, increasing the exploitation rate in this circumstances, the system tends to a fixed point, a constant that doesn't vary much, with the variation of the exploitation rate as a response to a null profit. This conclusion contradicts the hypothesis of the decreasing of the profit rate in time.

When the exploitation rate with null profit exceeds a critical point (in our case $a>12.5$ ), we have a bifurcation, and the system starts to present a cyclic oscillation between two profit rate values. According to the change of the parameter $a$, we obtain a bifurcation cascade, period duplication, that can be seen in the bifurcation diagram (figure 6). For certain values 
of $a$ the Lyapunov exponents, computed numerically (equation (4.2)), start to be positive, indicating a lack of stable orbits, the system doesn't tend to any orbits in the long term. We have chaos and total unpredictability of the system. When the exploitation rate in the origin is too high, the system starts to exhibit, again, stable orbits in a finite number (period five and three), but the Sharkovsky theorem [10] guarantees the existence of an infinite number of unstable orbits for each value of $a$. So there exists chaos and a large intrinsic complexity, justified by the fact that the topological entropy is positive (which implies chaos). There exists, in the last situation, a set of initial conditions, with total Lebesgue measure, that tends to those stable orbits. We can, in average, predict that the system will oscillate with a fixed period between five or three levels (which it also implies chaos in this circumstances [7]).

We observed that when the time unit of this iterated system becomes very large, decades for example, this modulation can explain phenomenons like the Kondratieff waves, by variations of the profit rate in time according to the Kondratieff undulatory law, that governs all of the economy. We also illustrated that Allee effect has a stabilisation effect of the profit rate, giving us information for the next results. In other words, the system becomes completely predictable with Allee effect.

Whatever the order of the magnitudes of $a$, when we use a discrete dynamical modulation, the profit rate doesn't necessarily fall to zero.

\section{References}

[1] K.M. Brooks, H. Bruin, Topics From One-Dimensional Dynamics, London Mathematical Society, Student Texts 62, Cambridge University Press, 2004.

[2] S. Elaydi, Discrete Chaos, Chapman and Hall/CRC, second Edition, 2008.

[3] S. Elaydi, An Introduction to Difference Equations, Springer, Third Edition, 2005.

[4] J. Guckenheimer, On bifurcation of maps of the interval, Invent. Math., Vol. 39 , pp. 165-178, 1977. 
[5] J. Li, B. Song and X. Wang, An extended discrete Ricker population model with Allee effects, Journal of Difference Equations and Applications, Vol. 13, No. 4, pp. 309-321, 2007.

[6] N. Kondratieff, The Long Waves in Economic Life, The Review of Economic Statistics, Vol. XVII, No. 6, pp. 105-115, 1935.

[7] T. Li, J. Yorke, Period Three Implies Chaos, Am. Math. Monthly, Vol. 82, pp. 985-992, 1975

[8] W. de Melo, S. van Strien, One-Dimensional Dynamics, Springer-Verlag, 1992.

[9] J. Milnor, W. Thurston, On iterated maps of the interval, Dynamical Systems: Proc. Univ. of Maryland, Lecture Notes in Mathematics vol. 1342, pp. 465-563, 1986-1987.

[10] A. Sharkovsky, Coexistence of cycles of a continuous mapping of the line into itself, Ukrain. Math. Zh., Vol. 16, pp. 61-71., 1964

[11] D. Singer, Stable orbits and bifurcation of maps of the interval, Siam J. Appl. Math., Vol. 35, No. 2, pp. 260-267, 1978.

[12] G. Statter, Tendência decrescente da taxa de lucro, Preprint, ISCTE, 2003. 